where $C$ is an arbitrary analytic Jordan curve, $z=\alpha$ is a point interior to $C, f(z)$ is of class $E_{p}$ interior to $C$, and $n(z)$ is the modulus on $C$ of a function $N(z)$ analytic and nonvanishing in the closed region $\Gamma$, is

$$
F_{0}(z)=A\left[\frac{N(\alpha)}{N(z)} \cdot \frac{g^{\prime}(z)}{g^{\prime}(\alpha)}\right]^{1 / p} \text {. }
$$

Let $P_{n}(z)$ be the corresponding minimizing polynomial of degree $n$. Then the sequence $P_{n}(z), n=0,1,2, \cdots$, converges maximally to $F_{0}(z)$ on $\Gamma$.

\title{
REFERENCES
}

1. Keldysch and Lavrentieff, Domaines limites par des courbes rectifiables, Ann. Ecole Norm. (3) vol. 54 (1937) pp. 1-38.

2. F. Riesz, Über die Randwerte einer analytischen Funktion, Math. Zeit. vol. 18 (1923) pp. 87-95.

3. J. L. Walsh, Interpolation and approximation by rational functions in the complex domain, Amer. Math. Soc. Colloquium Publications, vol. 20, 1935.

UNIVERSITY OF WISCONSIN

\section{NOTE ON THE LOCATION OF THE CRITICAL POINTS OF HARMONIC FUNCTIONS}

\section{J. L. WALSH}

The object of this note is to publish the statement of the following theorem.

THEOREM I. In the extended $(x, y)$-plane let $R_{0}$ be a simply-connected region bounded by a continuum $C_{0}$ not a single point, and let the disjoint continua $C_{1}, C_{2}, \cdots, C_{n}$ lie interior to $R_{0}$ and together with $C_{0}$ bound $a$ subregion $R$ of $R_{0}$. By means of a conformal map of $R_{0}$ onto the unit circle we define in $R_{0}$ non-euclidean lines, the images of arbitrary circles orthogonal to the unit circle. Denote by $\Pi$ the smallest closed non-euclidean convex region in $R_{0}$ which contains $C_{1}, C_{2}, \cdots, C_{n}$.

Let the function $u(x, y)$ be harmonic interior to $R$, continuous in the closure of $R$, with the values zero on $C_{0}$ and unity on $C_{1}, C_{2}, \cdots, C_{n}$. Then the critical points of $u(x, y)$ in $R$ are $n-1$ in number and lie in $I I$.

Critical points are of course to be counted according to their multiplicities.

A limiting case of Theorem I has already been established : ${ }^{1}$ if $f(z)$

Received by the editors November 29, 1945.

3 J. L. Walsh, Bull. Amer. Math. Soc. vol. 45 (1939) pp. 462-470; see p. 465. The result was proved later by W. Gontcharoff, C. R. (Doklady) Acad. Sci. URSS. vol. 36 (1942) pp. 39-41. 
is an analytic function whose modulus is constant on the boundary of a simply-connected region $R$, where $f(z)$ is analytic interior to $R$ and continuous in the closure of $R$, then the zeros of $f^{\prime}(z)$ in $R$ lie in the smallest non-euclidean convex polygon in $R$ containing the zeros of $f(z)$ in $R$. Theorem I is readily established by the use of this limiting case, and of methods developed elsewhere by the present writer; ${ }^{2}$ details are left to the reader.

Theorem I admits an extension to the case where $R_{0}$ is bounded by $C_{0}$, and the subregion $R$ of $R_{0}$ is bounded by $C_{0}$ and by further disjoint continua $C_{1}, C_{2}, \cdots, C_{m}, C_{m+1}, \cdots, C_{n}$ in $R_{0}$; the function $u(x, y)$ is supposed harmonic interior to $R$, continuous in the closure of $R$, with the values zero on $C_{0}$, unity on $C_{1}, C_{2}, \cdots, C_{m}$, and minus unity on $C_{m+1}, C_{m+2}, \cdots, C_{n}$; a non-euclidean line $\Lambda$ in $R_{0}$ (if existent) which separates $C_{1}, C_{2}, \cdots, C_{m}$ from $C_{m+1}, C_{m+2}, \cdots, C_{n}$ cannot pass through a critical point of $u(x, y)$. If a $\Lambda$ exists, the points of $R_{0}$ which do not lie on any such $\Lambda$ form two disjoint non-euclidean convex point sets in $R_{0}$ which are closed with respect to $R_{0}$, which contain respectively $C_{1}, C_{2}, \cdots, C_{m}$ and $C_{m+1}, C_{m+2}, \cdots, C_{n}$, and which together contain all critical points of $u(x, y)$ in $R$. This extension of Theorem I may likewise be proved from a limiting case already formulated (loc. cit.) for a region $R_{0}$ bounded by a circle.

\section{HARVARD UNIVERSITY}

2 Proc. Nat. Acad. Sci. U.S.A. vol. 20 (1934) pp. 551-554. 